Research Article

\title{
Nephroprotective Effects of Zataria multiflora Boiss. Hydroalcoholic Extract, Carvacrol, and Thymol on Kidney Toxicity Induced by Cisplatin in Rats
}

\author{
Esmaeel Panahi kokhdan (iD, ${ }^{1}$ Hossein Sadeghi ${ }^{D},{ }^{1}$ Shima Kazemi ${ }^{(D)}{ }^{2}$ \\ and Amir Hossein Doustimotlagh $\mathbb{D}^{1,3}$ \\ ${ }^{1}$ Medicinal Plants Research Center, Yasuj University of Medical Sciences, Yasuj, Iran \\ ${ }^{2}$ Student Research Committee, Yasuj University of Medical Sciences, Yasuj, Iran \\ ${ }^{3}$ Department of Clinical Biochemistry, Faculty of Medicine, Yasuj University of Medical Sciences, Yasuj, Iran \\ Correspondence should be addressed to Amir Hossein Doustimotlagh; amirhosseindoustimotlagh@gmail.com
}

Received 27 September 2020; Revised 5 January 2021; Accepted 17 January 2021; Published 27 January 2021

Academic Editor: Bashar Saad

Copyright (C) 2021 Esmaeel Panahi kokhdan et al. This is an open access article distributed under the Creative Commons Attribution License, which permits unrestricted use, distribution, and reproduction in any medium, provided the original work is properly cited.

\begin{abstract}
Background. Cisplatin (Cis) is an anticancer drug; however, it has dose-dependent renal toxicity. The current study aims to investigate the protective effects of Zataria multiflora Boiss. hydroalcoholic extract (Z.M.B), carvacrol, and thymol on cisplatininduced nephrotoxicity in rats. Materials and Methods. Forty-two Wistar male rats were randomly allocated into six groups $(n=7)$. Group I received normal saline; group II received Cis $(7 \mathrm{mg} / \mathrm{kg}$. ip); group III received the Z.M.B extract only $(500 \mathrm{mg} / \mathrm{kg} /$ d, po); group IV received Z.M.B extract $(500 \mathrm{mg} / \mathrm{kg} / \mathrm{d}$, po $)+$ Cis; group V received carvacrol $(50 \mathrm{mg} / \mathrm{kg} / \mathrm{d}$, po $)+$ Cis; and group VI received thymol $(50 \mathrm{mg} / \mathrm{kg} / \mathrm{d}, \mathrm{po})+$ Cis. The levels of biochemical markers, oxidative stress parameters, and histopathological staining were determined in serum and renal tissues. Also, the chemical compositions (carvacrol and thymol) of the Z.M.B extract were assayed by HPLC analysis. Result. The results revealed that Z.M.B extract, carvacrol, and thymol markedly decreased the renal index as compared with the Cis-only group. Also, carvacrol and thymol significantly reduced the blood urea nitrogen level as compared with the Cis-only group. Furthermore, Z.M.B extract, carvacrol, and thymol significantly attenuated the Cis-induced increase in malondialdehyde and nitric oxide metabolite. Additionally, histopathological examination showed that Z.M.B extract, carvacrol, and thymol markedly ameliorated Cis-induced renal tubular necrosis. Conclusion. The results showed renoprotective effects of Z.M.B extract, carvacrol, and thymol in Cis-induced nephrotoxicity in rats. Therefore, Z.M.B extract can be considered as a potential candidate for the protection of nephrotoxicity induced by Cis.
\end{abstract}

\section{Introduction}

Cisplatin (Cis) as the short form of cis-diaminedichloroplatinum is one of the major effective drugs used in chemotherapy for a variety of cancers including testicular, ovarian, and cervical cancers [1]. However, nephropathy is a serious complication of this drug, which makes it necessary to reduce the dose of this drug. A number of cases of acute renal failure in hospitalized patients are due to the unavoidable prescription of Cis [2], and despite the hydration therapy as a way to reduce renal toxicity, about one-third of patients who received Cis would experience irreversible kidney injuries [3]. The free Cis in the blood is easily filtered by glomeruli and enters the tubular cell through a transfer process. The main route for the transfer of Cis is active in renal transfer cells; although some amount of Cis also enters through the process of simple release, the kidney collects more Cis than any other organ and is the main route for its excretion [4]. Pathological changes in Cis-induced renal toxicity occur in proximal tubules because they accumulate the highest amount of Cis [5]. The intracellular effects of Cis include mitochondrial damage, cessation of the cell cycle, 
disruption of cellular transport systems, as well as induction of apoptosis or necrosis [6]. Cisplatin produces free radicals and activates the cascades of cell death signaling; then, it disrupts the antioxidant defense system [7]. Our previous study showed that antioxidant compounds such as Stachys pilifera Benth. extract had a protective role against cisplatininduced renal toxicity [8].

Medicinal plants or their active ingredients are widely used in the treatment of diseases. Several types of medicinal plants have been reported to reduce the toxicity of Cis [9]. Zataria multiflora Boiss. (Z.M.B) is a well-known medicinal plant of the Lamiaceae family. In traditional medicine, this plant is used as an antispasmodic and antitussive drug, as well as it is used in the treatment of lung infection and influenza. Carvacrol and thymol are among the important compounds in Z.M.B that are also are responsible for $70 \%$ of Z.M.B essential oil. The presence of these compounds in Z.M.B accounts for its high antioxidant properties [10, 11]. In addition, Z.M.B has shown significant protective and inhibitory properties against tissue damage caused by cellular oxidants [12]. Therefore, the current study was designed to investigate the effects of Z.M.B extract and its two main ingredients, carvacrol and thymol, on Cis-induced toxicity in rats.

\section{Materials and Methods}

2.1. Plant Materials. Zataria multiflora Boiss was collected in the spring of 2019 from Dena Mountain in Yasuj, Iran. The plant was approved by a botanist (herbarium number; Hyu-855-37687). Their aerial parts were dried in the shade away from direct sunlight. The leaves were separated from the stem and were ground and powdered.

2.2. Extract Preparation Method. The extraction was performed with maceration technique in which $200 \mathrm{~g}$ of plant leaf powder was soaked in $1 \mathrm{~L}$ of $75 \%$ ethanol at room temperature for 48 hours. This practice was repeated three times. The extract was concentrated in vacuum and dried at $40^{\circ} \mathrm{C}$ in an incubator. The dried extract was stored at $-20^{\circ} \mathrm{C}$ [13].

2.3. Animals. Forty-two rats with a weight range of 180 to $220 \mathrm{~g}$ were provided from the Pasteur Institute of Iran and were kept in 12 hours of light/12 hours of darkness and a temperature of $23 \pm 2$, and they were fed for a week. The study was approved by the Ethics Committee of the Yasuj University of Medical Sciences (Code: IR.YUMS.REC.1398.083). The animals were monitored according to the Principles of Laboratory Animal Care.

2.4. Experimental Design. Cisplatin was obtained from a Chinese pharmaceutical company named MYLAN (1 mg/ $\mathrm{mL}$ ). Carvacrol and thymol were purchased from Sigma Chemical Co. (St Louis, MO, USA) and Merck (Germany), respectively. Cisplatin was administered intraperitoneally at $7 \mathrm{mg} / \mathrm{kg}$ body weight on the first day [14]. The hydroalcoholic extract of Z.M.B was dissolved in sterile distilled water. Adult male rats were randomly assigned to six groups $(N=7)$ as follows:

Group I (normal): recipient of normal saline orally for 7 consecutive days

Group II (Cis): recipient of normal saline for 7 consecutive days after administration of Cis $(7 \mathrm{mg} / \mathrm{kg})$

Group III (500 mg/kg extract): recipient of only Z.M.B extract $(500 \mathrm{mg} / \mathrm{kg})$ orally for 7 consecutive days

Group IV (500 mg/kg extract + Cis): recipient of Z.M.B extracts $(500 \mathrm{mg} / \mathrm{kg})$ orally for 7 days after administration of Cis $(7 \mathrm{mg} / \mathrm{kg})$

Group V (carvacrol $50 \mathrm{mg} / \mathrm{kg}$ ): recipient of carvacrol $500 \mathrm{mg} / \mathrm{kg}$ orally for 7 days after administration of Cis $(7 \mathrm{mg} / \mathrm{kg})$

Group VI (thymol $50 \mathrm{mg} / \mathrm{kg}$ ): recipient of thymol $50 \mathrm{mg} / \mathrm{kg}$ orally 7 days after administration of Cis $(7 \mathrm{mg} / \mathrm{kg})$

On the eighth day of the study, rats were anesthetized with ether and blood was collected from their hearts to measure biochemical markers in the serum. Then, both kidneys were removed from the body and were washed with normal saline. One kidney was placed in $10 \%$ formalin for histopathological evaluation, and the other was homogenized in PBS $(10 \mathrm{mmol} / \mathrm{L}, \mathrm{pH} 7.4)$. Kidney homogenate was stored in a refrigerator at $-20^{\circ} \mathrm{C}$ to determine the levels of nitrite oxide (NO) metabolite, malondialdehyde (MDA), the ferric reducing antioxidant power (FRAP), and total thiol $(\mathrm{tSH})$. Changes in their weights were measured on the first and eighth day of the experiment.

2.5. Measurements of Biochemical Tests. The concentrations of blood urea nitrogen (BUN) and creatinine (Cr) were determined using commercial kits (Pars Azmoon, Iran).

2.6. Measurement of MDA. Malondialdehyde was determined according to the reaction with thiobarbituric acid (TBA) [15]. $250 \mu \mathrm{L}$ of tissue homogenate or serum was added in $1000 \mu \mathrm{L}$ of reagent (15\% w/v trichloroacetic acid, $0.25 \mathrm{~N} \mathrm{HCl}$, and $0.375 \% \mathrm{w} / \mathrm{v} \mathrm{TBA})$, and the optical density was assayed at $535 \mathrm{~nm}$.

2.7. Measurement of NO Metabolite. NO metabolite levels were determined in serum and kidney homogenate using the Griess reagent [8]. Sodium nitrite was used as a standard.

2.8. Measurement of Total Antioxidant Capacity Using the FRAP Method. This method was based on the ability of tissue homogenate in regeneration of ferric ion $\left(\mathrm{Fe}^{3+}\right)$ to ferrous ion $\left(\mathrm{Fe}^{2+}\right)$ in the presence of tripyridyl-s-triazine (TPTZ). The result was a blue complex TPTZ-Fe ${ }^{2+}$ with the maximum absorption of $593 \mathrm{~nm}$. $\mathrm{FeSO}_{4} \quad 7 \mathrm{H}_{2} \mathrm{O}$ $(0-1000 \mu \mathrm{mol} / \mathrm{L})$ was used as the standard [16]. 
2.9. Measurement of the $t S H$ Level. Total thiol content was determined using a colorimetric method with minor modifications [17]. $25 \mu \mathrm{L}$ of tissue homogenate was added to $150 \mu \mathrm{L}$ of Tris-EDTA reagent in a microtube. After that, $790 \mu \mathrm{L}$ of absolute methanol and $10 \mu \mathrm{L}$ of 5,5-dithio-bis- $(2-$ nitrobenzoic acid) were added. The tube was kept at $25^{\circ} \mathrm{C}$ for 15 minutes, and the absorption was measured at $412 \mathrm{~nm}$.

2.10. Histopathological Examinations. After removing the kidneys, the left kidney of each rat was cut in half. Then, the two halves were retained in the $10 \%$ formalin solution for a few days. After processing, the tissues were placed in the paraffin and cut into 3 to 4 micrometer slices. The incisions were placed on glass slides and stained with hematoxylin and eosin (H\&E) staining.

2.11. Chromatographic Conditions. High-performance liquid chromatography (HPLC) was performed on KNAUER liquid chromatography (Berlin, Zehlendorf, Germany) equipped with a micro vacuum degasser, a quaternary pump, a UV-VIS 2550 detector (was set at $220 \mathrm{~nm}$ for thymol and carvacrol) and a Zorbax SB-C 18 column $(250 \mathrm{~mm} \times 3.9 \mathrm{~mm}$ id, 5 micrometer particle size $)$, and an injection volume of $20 \mu \mathrm{L}$. The mobile phase contained acetonitrile-water mixture $(45: 55 \mathrm{~V} / \mathrm{V} \%)$ with a flow rate of $1.1 \mathrm{ml} \cdot \mathrm{min}^{-1}$.

2.12. Statistical Analysis. Data were evaluated using the oneway ANOVA test. Tukey's multiple comparison was used to express significant statistical significance. The data were presented as mean \pm SEM. $P \leq 0.05$ was considered statistically significant in all experiments.

\section{Results}

3.1. Body Weight Changes. In terms of body weight, the Cis group lost some weight in comparison with the control group (Table 1). However, Z.M.B and carvacrol to some extent reversed the weight loss as compared with Cis-only group.

3.2. Effects of Cisplatin on Biochemical Tests and Renal Index. Creatinine, BUN, and renal index in the Cis group indicated a marked increase as compared with the control group $(P \leq 0.05)$. The administration of hydroalcoholic extract of Z.M.B extract, carvacrol, and thymol significantly decreased the renal index in comparison with the Cis group $(P \leq 0.05)$. Treatment with carvacrol and thymol markedly decreased the BUN level as compared with the Cis group $(P \leq 0.05)$. However, the administration of hydroalcoholic extract of Z.M.B had no effect on BUN and $\mathrm{Cr}$ in comparison with the Cis group (Table 2).

3.3. Assessment of Renal Oxidative Stress Parameters. As indicated in Table 3, serum contents of MDA, FRAP, and NO metabolite markedly augmented in the Cis group in comparison with the control group $(P \leq 0.05)$, while treatment with hydroalcoholic extract of Z.M.B, carvacrol, and thymol significantly reduced serum levels of NO metabolite and MDA as compared with the Cis group. However, the FRAP content did not significantly change in the treated groups in comparison with the Cis group (Table 3).

The present study showed that $\mathrm{Cis}$ increased oxidative stress parameters such as NO metabolite and MDA, while it reduced the $\mathrm{tSH}$ level in renal tissue as compared with the control group (Table 4). The administration of Z.M.B extract, carvacrol, and thymol significantly reduced the NO metabolite level in comparison with the Cis group; however, only carvacrol and thymol significantly reduced the MDA level as compared with the Cis group. The $\mathrm{tSH}$ content in the treated groups did not change significantly compared with the Cis group (Table 4).

3.4. Histopathological Studies. According to Figure 1, histological results in the control group showed that the appearance of the kidneys, glomerulus, and urinary tubes were normal. In the Cis group, the size of the kidneys was larger, and the proximal, distal, and urethral tubules were dilated, indicating the destruction of glomeruli, necrosis of the urinary tract, and the infiltration of inflammatory cells. In the group that received both Z.M.B extract and Cis, the destruction of glomeruli decreased to some extent, the bleeding points were decreased, and the size of the kidneys was smaller than only the Cis group, but edema was observed. In the carvacrol and thymol groups, the damages caused by Cis were largely healed and were similar to those of the control group.

3.5. Chromatographic Analysis. The concentration of thymol and carvacrol compounds in Z.M.B extract was determined using the standard method. The calibration curve (Figure 2) was plotted according to the concentration (0.0005 to $\left.2 \mathrm{mg} \cdot \mathrm{L}^{-1}\right)$, and the unknown concentration of each analyte was calculated. Based on the chromatographic data and standard increase method, the amount of thymol and carvacrol in Z.M.B extract were 101.69 and $81.70 \mathrm{mg} \cdot \mathrm{g}^{-1}$, respectively (inset of Figure 2).

\section{Discussion}

The result of the present study revealed that the hydroalcoholic extract of Z.M.B and its two main ingredients, thymol and carvacrol, reduced the renal toxicity induced by Cis in rats. The main limiting factor for the clinical administration of Cis is its renal toxicity. Despite the constant control of serum concentrations during the administration of Cis, dose-dependent nephrotoxicity is still observed in patients $[18,19]$.

Cisplatin-induced nephrotoxicity appears mainly for at least 3 days after the beginning of treatment, and its specific effects comprise a decrease in the glomerular filtration rate, an increase in serum creatinine and BUN levels, and a decrease in the ability of the kidneys for urine concentration. In addition to all these, proteinuria and electrolyte imbalance also occur [20-24]. Currently, much research has been 
TABLE 1: Effects of Z.M.B on body weight in rats before and after treating with cisplatin (in grams).

\begin{tabular}{lccc}
\hline Treatment group & Before treatment $\left(W_{0}\right)$ & After treatment $\left(W_{1}\right)$ & $\%$ change $\left(W_{1}-W_{0} / W_{0}\right)$ \\
\hline Control & $221.00 \pm 23.18$ & $250.83 \pm 22.00$ & 13.50 \\
Cis & $186.67 \pm 18.78$ & $197.50 \pm 1.12$ & 5.80 \\
Z.M.B only & $190.00 \pm 12.91$ & $218.51 \pm 5.54$ & 15.00 \\
Z.M.B + Cis & $198.00 \pm 13.01$ & $216.33 \pm 13.21$ & 9.26 \\
Carvacrol + Cis & $165.00 \pm 6.06$ & $193.83 \pm 9.41$ & 17.47 \\
Thymol + Cis & $223.33 \pm 23.23$ & $239.50 \pm 14.59$ & 7.24 \\
\hline
\end{tabular}

Results are presented as mean \pm SEM. Cis: cisplatin; Z.M.B: hydroalcoholic extract of Zataria multiflora Boiss.

TABLE 2: Effects of Z.M.B hydroalcoholic extract, carvacrol, and thymol on kidney markers in Cis-induced toxicity in rats.

\begin{tabular}{|c|c|c|c|}
\hline Treatment group & BUN (mg/dL) & Creatinine $(\mathrm{mg} / \mathrm{dL})$ & Body weight/kidney weight index \\
\hline Control & $17.40 \pm 0.78$ & $0.48 \pm 0.02$ & $0.74 \pm 0.05$ \\
\hline Cis & $29.32 \pm 2.76^{* * *}$ & $0.61 \pm 0.03^{*}$ & $1.03 \pm 0.03^{* * *}$ \\
\hline Z.M.B only & $18.68 \pm 1.34$ & $0.49 \pm 0.02$ & $0.73 \pm 0.04$ \\
\hline Z.M.B + Cis & $22.52 \pm 2.26$ & $0.53 \pm 0.04$ & $0.84 \pm 0.03^{\#}$ \\
\hline Carvacrol + Cis & $20.95 \pm 1.89^{\#}$ & $0.5 \pm 0.02$ & $0.89 \pm 0.04^{\#}$ \\
\hline Thymol + Cis & $20.88 \pm 0.95^{\#}$ & $0.57 \pm 0.03$ & $0.80 \pm 0.03^{\# \#}$ \\
\hline
\end{tabular}

Values are presented as mean \pm SEM. Statistically significant compared with the control group, ${ }^{*} P \leq 0.05$ and ${ }^{* * *} P \leq 0.001$; statistically significant compared with the Cis group, ${ }^{\#} P \leq 0.05$ and ${ }^{\# \#} P \leq 0.01$. Cis: cisplatin; Z.M.B: hydroalcoholic extract of Zataria multiflora Boiss.

TABLE 3: Effects of Z.M.B hydroalcoholic extract, carvacrol, and thymol on the serum oxidative stress markers in Cis-induced toxicity in rats.

\begin{tabular}{lccc}
\hline Treatment group & MDA $(\mathrm{nmol} / \mathrm{L})$ & NO $(\mu \mathrm{mol} / \mathrm{L})$ & FRAP $(\mu \mathrm{mol} / \mathrm{L})$ \\
\hline Control & $487.17 \pm 12.21$ & $0.50 \pm 0.02$ & $305.10 \pm 34.07$ \\
Cis & $612.17 \pm 16.11^{*}$ & $1.20 \pm 0.12^{* * *}$ & $672.43 \pm 74.73^{*}$ \\
Z.M.B only & $554.82 \pm 14.91$ & $0.50 \pm 0.04$ & $361.87 \pm 47.77$ \\
Z.M.B + Cis & $443.38 \pm 52.17^{\# \#}$ & $0.59 \pm 0.09^{\# \#}$ & $492.75 \pm 72.29$ \\
Carvacrol + Cis & $419.87 \pm 29.92^{\# \# \#}$ & $0.48 \pm 0.05^{\# \# \#}$ & $566.68 \pm 143.76$ \\
Thymol + Cis & $449.59 \pm 11.43^{\#}$ & $0.57 \pm 0.04^{\# \#}$ & $610.35 \pm 62.38$ \\
\hline
\end{tabular}

Values are presented as mean \pm SEM. Statistically significant compared with the control group, ${ }^{*} P \leq 0.05$ and ${ }^{* * *} P \leq 0.001$; statistically significant compared with the Cis group, ${ }^{\#} P \leq 0.05,{ }^{\# \#} P \leq 0.01$, and ${ }^{\# \# \#} P \leq 0.001$. Cis: cisplatin; Z.M.B: hydroalcoholic extract of Zataria multiflora Boiss.

TABLE 4: Effects of Z.M.B hydroalcoholic extract, carvacrol, and thymol on the kidney tissue oxidative stress markers in Cis-induced toxicity in rats.

\begin{tabular}{lccc}
\hline Treatment group & MDA (nmol/g tissue) & NO $(\mu \mathrm{mol} / \mathrm{g}$ tissue $)$ & $\mathrm{tSH}(\mu \mathrm{mol} / \mathrm{g}$ tissue $)$ \\
\hline Control & $71.64 \pm 0.91$ & $0.35 \pm 0.06$ & $17.30 \pm 1.68$ \\
Cis & $84 \pm 1.33^{* *}$ & $0.64 \pm 0.02^{* * *}$ & $10.03 \pm 0.24^{* * *}$ \\
Z.M.B only & $77.88 \pm 3.20$ & $0.31 \pm 0.04$ & $13.36 \pm 0.95$ \\
Z.M.B + Cis & $76.28 \pm 1.98$ & $0.34 \pm 0.03^{\# \# \#}$ & $11.28 \pm 0.62$ \\
Carvacrol + Cis & $72.97 \pm 0.94^{\#}$ & $0.39 \pm 0.03^{\# \#}$ & $9.85 \pm 0.97$ \\
Thymol + Cis & $72.97 \pm 2.81^{\#}$ & $0.37 \pm 0.03^{\# \# \#}$ & $12.20 \pm 1.53$ \\
\hline
\end{tabular}

Values are presented as mean \pm SEM. Statistically significant compared with the control group, ${ }^{*} P \leq 0.05$ and ${ }^{* * *} P \leq 0.001$; statistically significant compared with the Cis group, ${ }^{\#} P \leq 0.05,{ }^{\# \#} P \leq 0.01$, and ${ }^{\# \# \#} P \leq 0.001$. Cis: cisplatin; Z.M.B: hydroalcoholic extract of Zataria multiflora Boiss.

done to understand the possible protective mechanisms that work against Cis toxicity and attempts have been made to clarify how it works [25].

Reactive oxygen species (ROSs) play a vital role in causing acute renal failure due to the administration of Cis [26]. It is not surprising; therefore, many antioxidants, such as vitamins $\mathrm{C}$ and $\mathrm{E}$, can reduce $\mathrm{Cis}$-induced nephrotoxicity [27]. In addition, $\mathrm{N}$-acetyl cysteine has been shown to reduce renal toxicity of Cis in laboratory animals and humans [28].

Medicinal plants show favorable effects against different nephrotoxic agents. Z.M.B extract contains known antioxidants such as carvacrol, thymol, and menthol. It seems that the antioxidants in Z.M.B (carvacrol and thymol) have been able to reduce the relative impairment of kidney function by reducing the amounts of ROS. Our research has shown that rats' weights reduced in the Cis group in comparison with the control group. However, the hydroalcoholic extract of Z.M.B, and carvacrol to some extent, reversed the weight loss caused by Cis.

Our findings confirmed the administration of cisplatin (7 mg/kg body weight) leads to acute renal failure by increasing kidney function tests and histological findings.

The nephroprotective effect of Z.M.B may be related to its inhibitory activity on the secretion or function of 


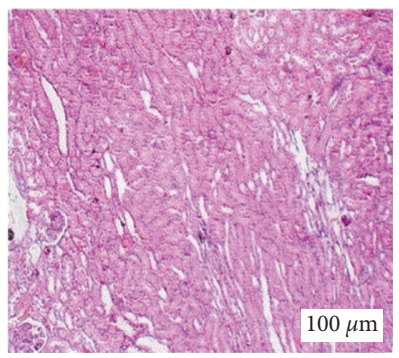

(a)

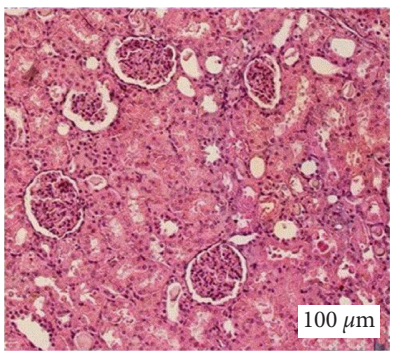

(d)

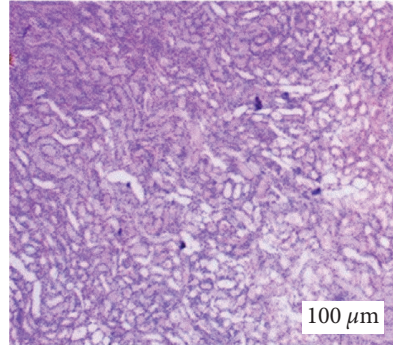

(b)

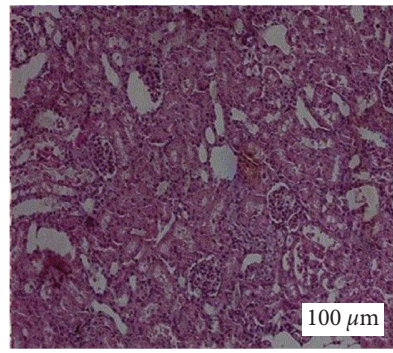

(e)

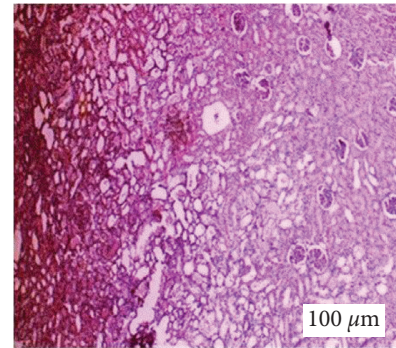

(c)

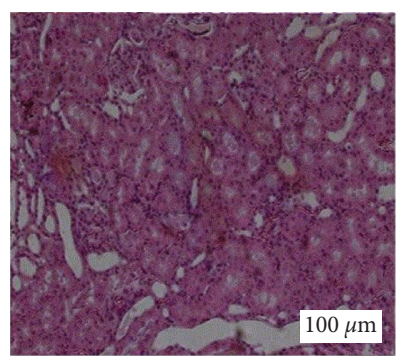

(f)

Figure 1: Histological changes in the renal tissue: (a) control, (b) cisplatin (Cis), (c) Zataria multiflora Boiss. extract (Z.M.B) only, (d) Z.M.B + Cis, (e) carvacrol + Cis, and (f) thymol + Cis (hematoxylin and eosin, $\times 100)$.

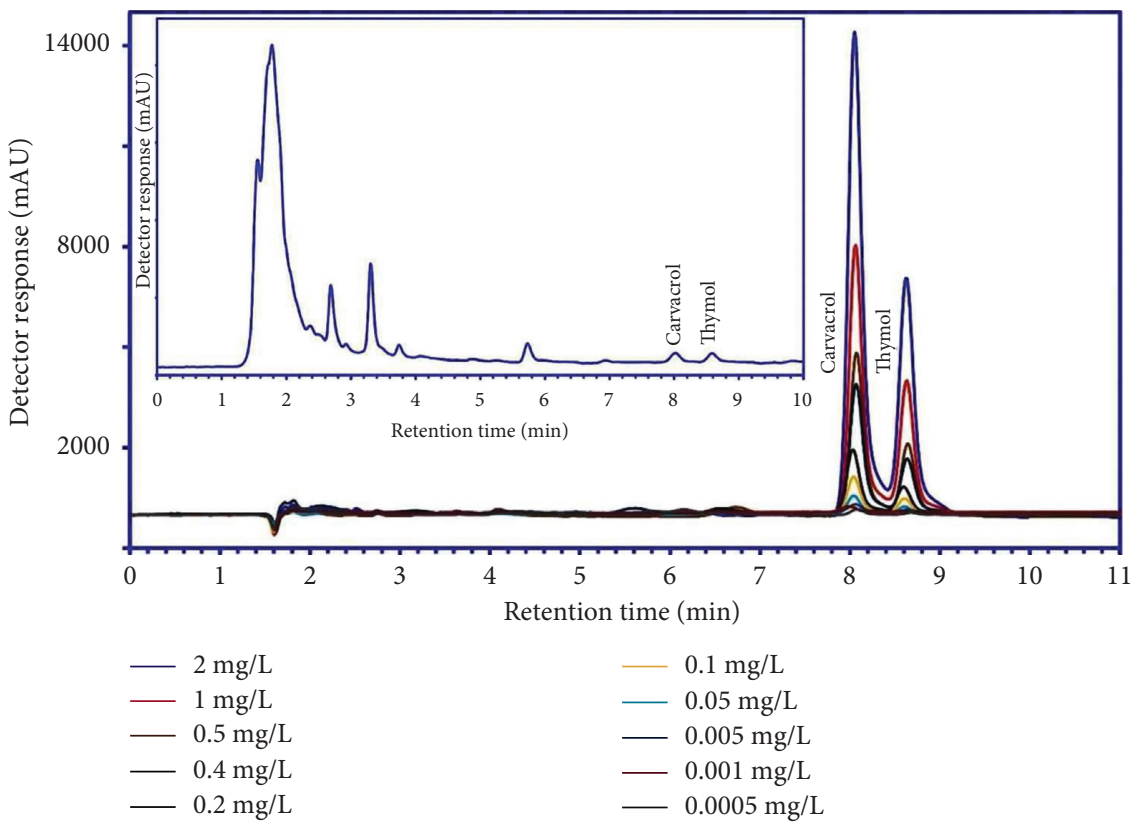

Figure 2: Chromatogram standard samples of thymol and carvacrol in distilled water at different concentrations and the hydroalcoholic extract of Zataria multiflora Boiss (inset in the figure).

inflammatory cytokines and pro-oxidants, which are involved in the damage of glomerular filtration rate [29]. Ibrahim et al. showed that a covalent bond is formed between platinum and some DNA bases, which can lead to cytotoxicity [30].

The present work indicated that the injection of Cis markedly increased the levels of MDA and NO metabolite in renal tissue and serum as compared with the control group. Several investigations have reported that Cis caused a significant elevation in the kidney MDA and NO metabolite concentration [31, 32]. Indeed, the increase of MDA and NO metabolite in the Cis group was lowered by Z.M.B, carvacrol, and thymol. Therefore, the beneficial action of Z.M.B, carvacrol, and thymol on MDA and NO metabolite contents might be due to its antioxidant effects.

HPLC analysis indicated that Z.M.B extract contains phenolic compounds such as thymol and carvacrol. It seems that these phenolic compounds protect tissue against 
oxidative stress by scavenging free radicals, reducing NO metabolite, and inhibiting lipid peroxidation.

In the current study, tSH groups were reduced following Cis administration. Z.M.B- and thymol-treated rats showed higher tSH levels rather than Cis-only group, demonstrating that Z.M.B and thymol insignificantly accelerated the replenishing of the total thiol pool. The effect of Z.M.B and thymol on total thiol contents may be attributed to its direct antioxidant activity [33].

\section{Conclusion}

The present study supports the role of oxidative stress in the pathophysiology of Cis-induced nephrotoxicity. Hydroalcoholic extract of Z.M.B and its two main ingredients (carvacrol and thymol) were able to improve oxidative stress and kidney damage caused by Cis. However, more studies are required to clarify mechanisms involved in Z.M.B action on Cis-induced nephrotoxicity.

\section{Data Availability}

The data supporting the findings of this study are available within the article.

\section{Disclosure}

This study was part of a MD thesis.

\section{Conflicts of Interest}

The authors declare that they have no conflicts of interest.

\section{Acknowledgments}

This study was financially supported by the Research Council of the Yasuj University of Medical Sciences, Yasuj, Iran.

\section{References}

[1] P. G. Rose, S. Ali, E. Watkins et al., "Long-term follow-up of a randomized trial comparing concurrent single agent cisplatin, cisplatin-based combination chemotherapy, or hydroxyurea during pelvic irradiation for locally advanced cervical cancer: a gynecologic oncology group study," Journal of Clinical Oncology, vol. 25, no. 19, pp. 2804-2810, 2007.

[2] J. S. Berns and P. A. Ford, "Renal toxicities of antineoplastic drugs and bone marrow transplantation," Seminars in $\mathrm{Ne}$ phrology, vol. 17, no. 1, pp. 54-66, 1997.

[3] J. T. Santoso, J. A. Lucci, R. L. Coleman, I. Schafer, and E. V. Hannigan, "Saline, mannitol, and furosemide hydration in acute cisplatin nephrotoxicity: a randomized trial," Cancer Chemotherapy and Pharmacology, vol. 52, no. 1, pp. 13-18, 2003.

[4] M. Sooriyaarachchi, A. Narendran, and J. Gailer, "Comparative hydrolysis and plasma protein binding of cis-platin and carboplatin in human plasma in vitro," Metallomics, vol. 3, no. 1, pp. 49-55, 2011.

[5] G. Ciarimboli, "Membrane transporters as mediators of cisplatin side-effects," Anticancer Research, vol. 34, no. 1, pp. 547-550, 2014.
[6] Y. I. Chirino and J. Pedraza-Chaverri, "Role of oxidative and nitrosative stress in cisplatin-induced nephrotoxicity," $E x$ perimental and Toxicologic Pathology: Official Journal of the Gesellschaft Fur Toxikologische Pathologie, vol. 61, no. 3, pp. 223-242, 2009.

[7] I. Arany, J. K. Megyesi, H. Kaneto, P. M. Price, and R. L. Safirstein, "Cisplatin-induced cell death is EGFR/src/ ERK signaling dependent in mouse proximal tubule cells," American Journal of Physiology-Renal Physiology, vol. 287, no. 3, pp. F543-F549, 2004.

[8] H. Sadeghi, M. Mansourian, E. Panahi kokhdan et al., “Antioxidant and protective effect of Stachys pilifera Benth. against nephrotoxicity induced by cisplatin in rats," Journal of Food Biochemistry, vol. 44, no. 5, Article ID e13190, 2020.

[9] Y. Shimeda, Y. Hirotani, Y. Akimoto et al., "Protective effects of capsaicin against cisplatin-induced nephrotoxicity in rats," Biological \& Pharmaceutical Bulletin, vol. 28, no. 9, pp. 1635-1638, 2005.

[10] F. Fatemi, Y. Asri, I. Rasooli, S. D. Alipoor, and M. Shaterloo, "Chemical composition and antioxidant properties of $\gamma$-irradiated Iranian Zataria multiflora extracts," Pharmaceutical Biology, vol. 50, no. 2, pp. 232-238, 2012.

[11] A. R. Khosravi, H. Shokri, Z. Tootian, M. Alizadeh, and R. Yahyaraeyat, "Comparative efficacies of Zataria multiflora essential oil and itraconazole against disseminated Candida albicans infection in BALB/c mice," Brazilian Journal of Microbiology, vol. 40, no. 3, pp. 439-445, 2009.

[12] H. Kazemian, H. Heidari, J. K. Yamchi et al., "In vitro antimycobacterial activity of three medicinal plants of Lamiaceae family," Recent Patents on Anti-infective Drug Discovery, vol. 13, no. 3, pp. 240-245, 2018.

[13] M. Mansourian, A. Mirzaei, N. Azarmehr, H. Vakilpour, E. P. Kokhdan, and A. H. Doustimotlagh, "Hepatoprotective and antioxidant activity of hydroalcoholic extract of Stachys pilifera Benth. on acetaminophen-induced liver toxicity in male rats," Heliyon, vol. 5, no. 12, Article ID e03029, 2019.

[14] A. Atessahin, S. Yilmaz, I. Karahan, A. O. Ceribasi, and A. Karaoglu, "Effects of lycopene against cisplatin-induced nephrotoxicity and oxidative stress in rats," Toxicology, vol. 212, no. 2-3, pp. 116-123, 2005.

[15] H. Ohkawa, N. Ohishi, and K. Yagi, "Assay for lipid peroxides in animal tissues by thiobarbituric acid reaction," Analytical Biochemistry, vol. 95, no. 2, pp. 351-358, 1979.

[16] H. Sadeghi, N. Azarmehr, F. Razmkhah et al., "The hydroalcoholic extract of watercress attenuates protein oxidation, oxidative stress, and liver damage after bile duct ligation in rats," Journal of Cellular Biochemistry, vol. 120, no. 9, 2019.

[17] N. Azarmehr, P. Afshar, M. Moradi et al., "Hepatoprotective and antioxidant activity of watercress extract on acetaminophen-induced hepatotoxicity in rats," Heliyon, vol. 5, no. 7, Article ID e02072, 2019.

[18] D. Beauchamp, P. Gourde, and M. G. Bergeron, "Subcellular distribution of gentamicin in proximal tubular cells, determined by immunogold labeling," Antimicrobial Agents and Chemotherapy, vol. 35, no. 11, pp. 2173-2179, 1991.

[19] G. R. Matzke, R. L. Lucarotti, and H. S. Shapiro, "Controlled comparison of gentamicin and tobramycin nephrotoxicity," American Journal of Nephrology, vol. 3, no. 1, pp. 11-17, 1983.

[20] N. Eisenberg and P. A. Miller, "The relation of empathy to prosocial and related behaviors," Psychological Bulletin, vol. 101, no. 1, p. 91, 1987.

[21] J. M. Eisenberg, H. Koffer, H. A. Glick et al., "What is the cost of nephrotoxicity associated with aminoglycosides?" Annals of Internal Medicine, vol. 107, no. 6, pp. 900-909, 1987. 
[22] H. Osswald, G. Nabakowski, and H. Hermes, "Adenosine as a possible mediator of metabolic control of glomerular filtration rate," Biochemical Aspects of Renal Function, vol. 12, no. 1-2, pp. 263-267, 1980.

[23] J. Fabre, J. Fillastre, J. Morin, and M. Rudhardt, "Nephrotoxicity of gentamicin," in Toxic NephropathiesKarger Publishers, Berlin, Germany, 1978.

[24] F. G. Falco, H. M. Smith, and G. M. Arcieri, "Nephrotoxicity of aminoglycosides and gentamicin," Journal of Infectious Diseases, vol. 119, no. 4-5, pp. 406-409, 1969.

[25] A. A. Fouad, M. A. Morsy, and W. Gomaa, "Protective effect of carnosine against cisplatin-induced nephrotoxicity in mice," Environmental Toxicology and Pharmacology, vol. 25, no. 3, pp. 292-297, 2008.

[26] K. K. Soni, H. K. Kim, B. R. Choi et al., "Dose-dependent effects of cisplatin on the severity of testicular injury in sprague dawley rats: reactive oxygen species and endoplasmic reticulum stress," Drug Design, Development and Therapy, vol. 10, pp. 3959-3968, 2016.

[27] B. H. Ali and M. S. Al Moundhri, "Agents ameliorating or augmenting the nephrotoxicity of cisplatin and other platinum compounds: a review of some recent research," Food and Chemical Toxicology, vol. 44, no. 8, pp. 1173-1183, 2006.

[28] D. Appenroth, K. Winnefeld, H. Schröter, and M. Rost, "Beneficial effect of acetylcysteine on cisplatin nephrotoxicity in rats," Journal of Applied Toxicology, vol. 13, no. 3, pp. 189-192, 1993.

[29] S. Cuzzocrea, E. Mazzon, L. Dugo et al., "A role for superoxide in gentamicin-mediated nephropathy in rats," European Journal of Pharmacology, vol. 450, no. 1, pp. 67-76, 2002.

[30] A. Ibrahim, M. A. A. Eldaim, and M. M. Abdel-Daim, "Nephroprotective effect of bee honey and royal jelly against subchronic cisplatin toxicity in rats," Cytotechnology, vol. 68, no. 4, pp. 1039-1048, 2016.

[31] F. Dehnamaki, A. Karimi, A. A. Pilevarian et al., "Treatment with troxerutin protects against cisplatin-induced kidney injury in mice," Acta Chirurgica Belgica, vol. 119, no. 1, pp. 31-37, 2019.

[32] E. H. Bae, J. Lee, S. K. Ma et al., "Lipoic acid prevents cisplatininduced acute kidney injury in rats," Nephrology Dialysis Transplantation, vol. 24, no. 9, pp. 2692-2700, 2009.

[33] T. Ochiai, S. Ohno, S. Soeda, H. Tanaka, Y. Shoyama, and H. Shimeno, "Crocin prevents the death of rat pheochromyctoma (PC-12) cells by its antioxidant effects stronger than those of $\alpha$-tocopherol," Neuroscience Letters, vol. 362, no. 1, pp. 61-64, 2004. 